\title{
Detailed characterization of log-periodic oscillations for an aperiodic Ising model
}

\author{
R. F. S. Andrade \\ Instituto de Física, Universidade Federal da Bahia, Campus da Federação 40210-340 Salvador, Brazil
}

(Received 19 November 1999)

\begin{abstract}
This work analyzes the behavior of Ising spins on a hierarchical lattice subject to relevant fluctuations on the coupling constants induced by a deterministic aperiodic sequence. The thermodynamical functions are evaluated within the method of transfer matrices. It is shown that log-periodic oscillations in the reduced temperature are present for all thermodynamical functions close to the critical point and that the nature of oscillations changes discontinuously at $T_{c}$ : for $T>T_{c}$ they are almost pure sinusoidal, while, for $T<T_{c}$, a second harmonic to the fundamental frequency is clearly observed.
\end{abstract}

PACS number(s): 05.50.+q, 64.60.Ak, 61.44.Br

Magnetic systems with lack of translational symmetry mediated by deterministic aperiodic sequences have been intensively investigated in recent years [1]. Such sequences, generated by substitution or inflation rules, have many of their properties well characterized from the mathematical point of view [2,3]. The presence of aperiodicity (either deterministic or random) may induce changes in the critical behavior of the originally uniform model. This occurs when the fluctuations in the coupling constants and fields are relevant or marginal according to the Luck criterion [4], that has been adapted [5-7] to analyze models defined on hierarchical lattices [8-12]. This extension is quite relevant, as these exact scale invariant lattices have shown to be appropriate for the analysis of the effect of deterministic aperiodicity: they lead to phase transitions at finite temperature; they can be exactly investigated with the help of renormalization procedures; it has been possible to choose an appropriate inflation rule for the aperiodicity which matches with the same invariance dictated by the geometry of the lattice.

Changes produced by relevant fluctuations include a drastic weakening of the criticality reflected in the new values for the critical exponents. Other important change is the emergence of log-periodic oscillations in the thermodynamical properties as function of the reduced temperature $[13,14]$. These oscillations are generally regarded as a consequence of a discrete scale invariance. However, it seems that they can only be observed if relevant fluctuations weakens the original robust transition.

In this work we present a detailed characterization of logperiodic oscillations for the Ising model on a hierarchical lattice with relevant fluctuations [15]. Our very precise results are based on the numerical iteration of a set of exact maps, the derivation of which requires no approximation and proceed within a transfer matrix (TM) formalism [16]. We show that oscillations are present in all thermodynamical functions for both $T<T_{c}$ and $T>T_{c}$, and not only for the specific heat $\left(T>T_{c}\right)$ as shown before [15]. The oscillatory behavior has been observed for values of the reduced temperature $t=\left|T-T_{c}\right| / T_{c}$ as small as $10^{-11}$. Also we present strong evidences that the periodic dependence changes at $T_{c}$ : For $T>T_{c}$, they can be fitted by one single sinusoidal function, while the presence of the second harmonic of the fundamental frequency is required for their description when $T<T_{c}$. We also show that the period of oscillations is well explained by a recently proposed Migdal-Kadanof renormalization group (MKRG) framework to account for a class of aperiodic models [17]. Finally, estimates for the critical exponents [15] have been improved.

We consider an aperiodic $\sigma_{i}= \pm 1$ Ising model on a hierarchical lattice, where any bond of a given generation is substituted by a set of $q=3$ parallel branches, each one containing a series of $p=3$ bonds. The formal Hamiltonian for the system is

$$
H=-\sum_{(i, j)} J_{i j} \sigma_{i} \sigma_{j}-h \sum_{i} \sigma_{i}
$$

where the first sum is performed over pairs $(i, j)$ of first neighbor sites, and $h$ is an uniform field acting on all sites of the lattice. The bonds $J_{i j}$ are the same on all parallel paths linking the two root points. They assume only two distinct values $J_{A}$ and $J_{B}$, according to the substitution rule for the sequence formed by the symbols $A$ and $B$ as $(A, B)$ $\rightarrow\left(A B^{2}, A^{3}\right)$.

MKRG analysis of the model shows that the usual saddle point related to the criticality of the uniform model becomes a full unstable node when $J_{A} \neq J_{B}$, and that the aperiodic

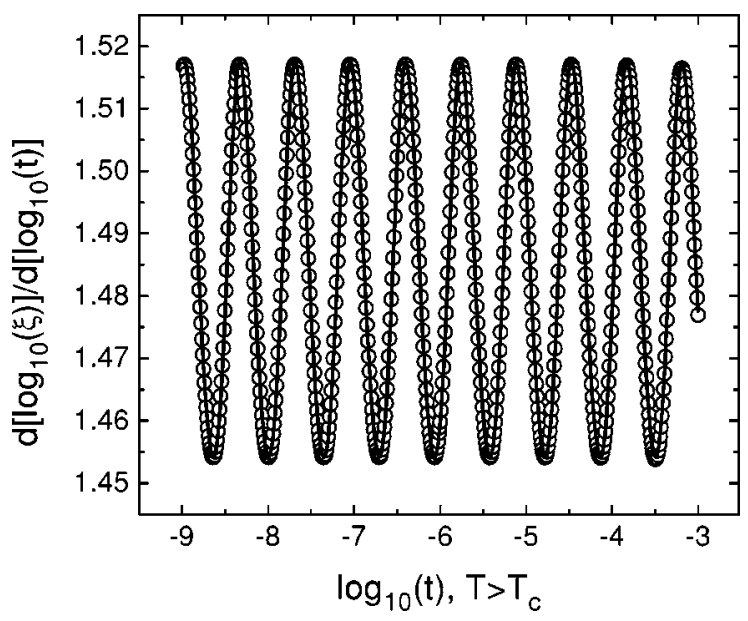

FIG. 1. Curve for $d\left(\log _{10} \xi\right) / d\left(\log _{10} t\right)$ vs $\log _{10} t\left(T>T_{c}\right)$ showing almost pure sinusoidal oscillations. For this and following figures open circles are evaluated from the set of maps, while full line corresponds to the fitting function. 


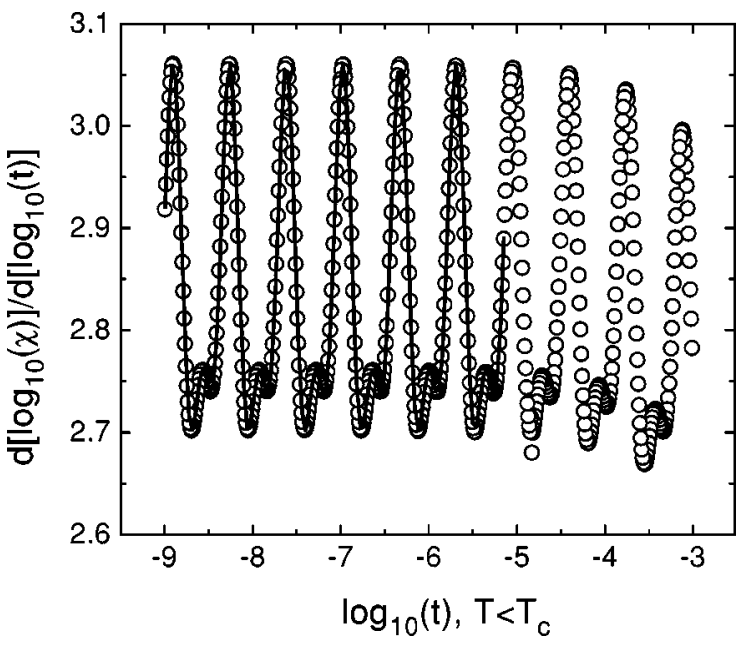

FIG. 2. Same as in Fig. 1 for the susceptibility $\chi$ and $T<T_{c}$. The presence of a second harmonic to the fundamental frequency is easily recognizable.

system does not belong to the same universality class of its uniform counterpart $[5,6]$. This has been confirmed by the numerical evaluation of the thermodynamical functions within the TM scheme, where new values for the critical exponents have been evaluated.

In a recent work, Haddad et al. [17] have shown that the second iterate of the MKRG transformation admits a pair of fixed saddle points with largest (unstable) eigenvalue $\Lambda_{c i c}$. Adapting the usual scaling MKRG arguments, they linked the phase transition of the aperiodic model to the period-2 cycle by

$$
\alpha=2-2 \frac{\ln b^{D}}{\Lambda_{\text {cic }}} .
$$

Moreover, they also show that, close to the fixed point, the specific free energy satisfies also the universal form

$$
f(x)=\left|x-x_{c}\right|^{2-\alpha} P\left(\frac{\ln \left|x-x_{c}\right|}{\ln \Lambda_{c i c}}\right),
$$

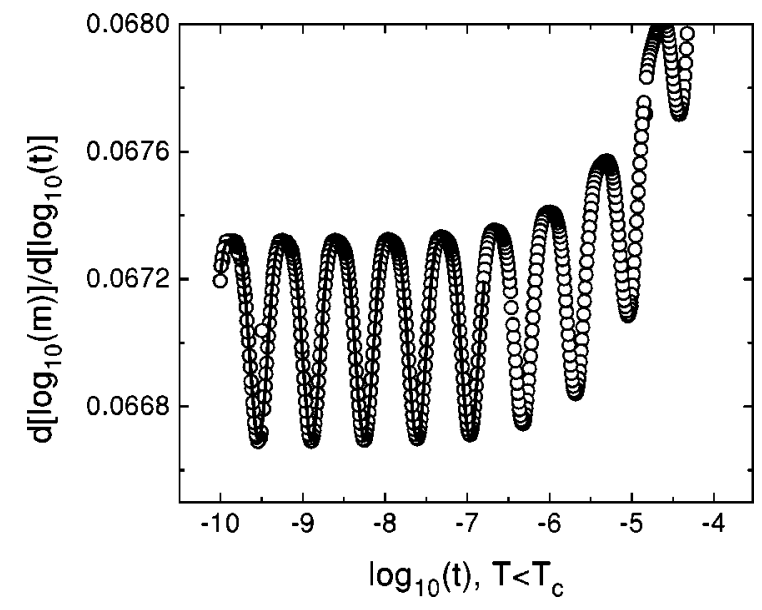

FIG. 3. The magnetization $m$ also entails the presence of a second harmonic, but its effect is less noticeable than that in Fig. 2.
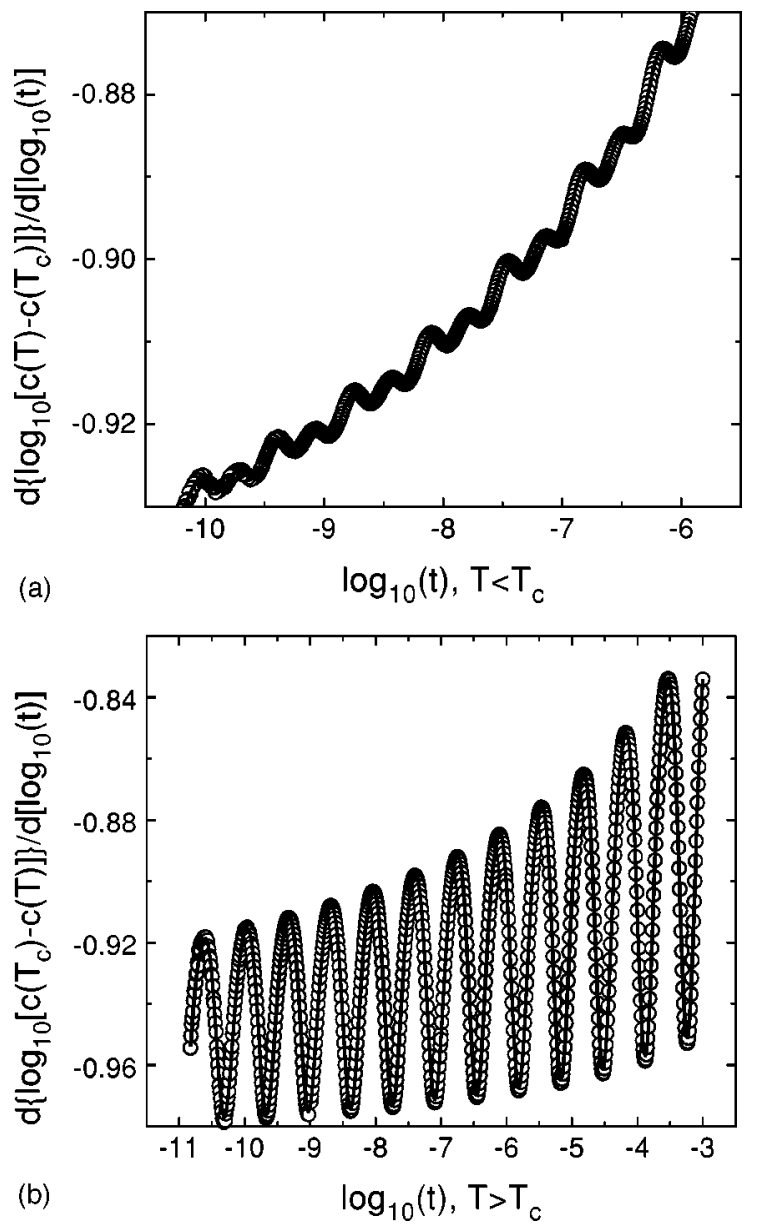

FIG. 4. Curves for $d\left[\log _{10}\left|c(T)-c\left(T_{c}\right)\right|\right] / d\left(\log _{10} t\right)$ vs $\log _{10} t$ for both $T<T_{c}$ (a) and $T>T_{c}$ (b). Note in (a) the presence of two harmonic contributions and a very small scaling region due to the presence of the maximum at $T_{m}$. When $T>T_{c}$ the curve shows almost pure harmonic oscillations with slightly decreasing amplitude.

where $x_{c}$ indicates the coordinates of any of the two points of the cycle and $P$ indicates an arbitrary period one function. So, log-periodic oscillations fit well into the general solution for the free energy. In fact, expression (3) represents the general solution within the MKRG scheme for the free energy for any model whose criticality is described by an usual single saddle point [18]. In most of the situations where an uniform model is considered, however, it is found that the arbitrary function $P$ reduces to a constant. This is not the case for a number of models with relevant aperiodic fluctuations, where the evaluation of the thermodynamical functions indicates the presence of oscillatory behavior. We show now that oscillations for the present model fit well into the general properties of Eq. (3).

The TM scheme used in our calculations has been detailed elsewhere [15], and we skip the derivation and the list of the maps used for the description of the thermodynamical properties of the model. They express the pertinent quantities of a given generation $G+1$ of the model in terms of those of the preceding generation $G$. The maps are written in terms of the free energy per spin $f_{G}$ and the correlation length $\xi_{G}$, defined as 
TABLE I. Values for the parameters of the fitting functions [Eqs. (5) and (7)] obtained for $\xi, \chi, m$, and $c$.

\begin{tabular}{|c|c|c|c|c|c|c|c|c|c|c|c|}
\hline$y$ & $T$ & $a$ & $\omega$ & $b_{1}$ & $b_{2}$ & $\phi_{1}$ & $\phi_{2}$ & $c$ & $d$ & $f$ & $\chi^{2}$ \\
\hline$\xi$ & $>T_{c}$ & $\begin{array}{c}1.48432 \\
\pm 0.00029\end{array}$ & $\begin{array}{c}9.77151 \\
\pm 0.00757\end{array}$ & $\begin{array}{c}0.03181 \\
\pm 0.00041\end{array}$ & & $\begin{array}{l}-0.34551 \\
\pm 0.04579\end{array}$ & & & & & $2.6 \times 10^{-6}$ \\
\hline$\xi$ & $>T_{c}$ & $\begin{array}{c}1.48431 \\
\pm 0.00015\end{array}$ & $\begin{array}{c}9.77480 \\
\pm 0.00401\end{array}$ & $\begin{array}{c}0.03157 \\
\pm 0.00021\end{array}$ & $\begin{array}{c}0.00187 \\
\pm 0.00021\end{array}$ & $\begin{array}{l}-0.33210 \\
\pm 0.02431\end{array}$ & $\begin{array}{l}-0.06751 \\
\pm 0.12825\end{array}$ & & & & $6.65 \times 10^{-7}$ \\
\hline$\chi$ & $<T_{c}$ & $\begin{array}{c}2.83366 \\
\pm 0.00027\end{array}$ & $\begin{array}{c}9.77179 \\
\pm 0.00175\end{array}$ & $\begin{array}{l}-0.15147 \\
\pm 0.00038\end{array}$ & $\begin{array}{l}-0.07676 \\
\pm 0.00004\end{array}$ & $\begin{array}{c}2.28748 \\
\pm 0.01229\end{array}$ & $\begin{array}{l}1.15802 \\
\pm 0.0232\end{array}$ & & & & $2.17 \times 10^{-6}$ \\
\hline$m$ & $<T_{c}$ & $\begin{array}{c}0.06708 \\
\pm 1.76 \times 10^{-6}\end{array}$ & $\begin{array}{c}9.77508 \\
\pm 0.00901\end{array}$ & $\begin{array}{l}-3.11 \times 10^{-4} \\
\pm 2.5 \times 10^{-6}\end{array}$ & $\begin{array}{l}-7.42 \times 10^{-5} \\
\pm 2.5 \times 10^{-6}\end{array}$ & $\begin{array}{l}-0.99609 \\
\pm 0.07275\end{array}$ & $\begin{array}{l}-2.09413 \\
\pm 0.13596\end{array}$ & & & & $8.92 \times 10^{-11}$ \\
\hline$c$ & $<T_{c}$ & $\begin{array}{l}-0.96514 \\
\pm 0.00053\end{array}$ & $\begin{array}{c}9.77426 \\
\pm 0.01047\end{array}$ & $\begin{array}{l}-0.01220 \\
\pm 0.00240\end{array}$ & $\begin{array}{c}0.01787 \\
\pm 0.00345\end{array}$ & $\begin{array}{c}0.45163 \\
\pm 0.08322\end{array}$ & $\begin{array}{c}1.68248 \\
\pm 0.14693\end{array}$ & $\begin{array}{c}2.21371 \\
\pm 0.05137\end{array}$ & $\begin{array}{c}1.77508 \\
\pm 0.01591\end{array}$ & $\begin{array}{c}1.23587 \\
\pm 0.09609\end{array}$ & $1.09 \times 10^{-8}$ \\
\hline$c$ & $>T_{c}$ & $\begin{array}{l}-0.99074 \\
\pm 0.00221\end{array}$ & $\begin{array}{c}9.77317 \\
\pm 0.00122\end{array}$ & $\begin{array}{l}-0.13533 \\
\pm 0.00155\end{array}$ & $\begin{array}{l}-0.01704 \\
\pm 0.00039\end{array}$ & $\begin{array}{c}0.16009 \\
\pm 0.00757\end{array}$ & $\begin{array}{c}0.7440 \\
\pm 0.02448\end{array}$ & $\begin{array}{c}0.26227 \\
\pm 0.00234\end{array}$ & $\begin{array}{c}0.73738 \\
\pm 0.02258\end{array}$ & $\begin{array}{c}0.64997 \\
\pm 0.00661\end{array}$ & $1.73 \times 10^{-7}$ \\
\hline
\end{tabular}

$$
\begin{gathered}
f_{G}=-\frac{T}{N_{G}} \ln \eta_{G}, \\
\xi_{G}=3^{G} / \ln \left(\eta_{G} / \epsilon_{G}\right) .
\end{gathered}
$$

$\eta_{G}$ and $\epsilon_{G}$ are the eigenvalues of the matrix $T_{G}$ that describes the interaction between the two root sites of the lattice, and $N_{G}=\left(5+3 \times 9^{G}\right) / 4$ counts the number of sites of the lattice in the generation $G$. These two variables are sufficient for the complete description of the homogeneous model when $h=0$. For the aperiodic case and when $h \neq 0$, a larger number of variables, defined in a similar way as Eq. (4), is required. The maps are iterated until convergence (relative precision of $10^{-16}$ ) is obtained. Temperature dependent initial conditions are required for the numeric process as well.

The criticality of the uniform model is characterized by a cusp singularity in the specific heat $c$, a vanishing spontaneous magnetization $m$ and divergences in the correlation length $\xi$ and susceptibility $\chi$. The critical exponents $\alpha$ $=-0.701, \beta=0.168, \gamma=2.356$, and $\nu=1.354$ have been evaluated within the TM method and satisfy the Rushbrook and hyperscale relations to a high precision, with errors less than $0.3 \%$.

When $J_{A} \neq J_{B}$ the system evolves into a universality class with completely different features. (i) The cusp for $c$ at $T_{c}$ disappears, replaced by a smooth maximum at $T_{m}$ slightly smaller than $T_{c}$ along with a critical behavior at $T_{c}$. (ii) The critical exponents assume universal values for all $J_{A} \neq J_{B}$, even for $J_{B}<0$. (iii) Presence of log-periodic behavior, as will be detailed with the help of Figs. 1-4. There we draw the derivatives of the decimal logarithm of $\xi, \chi, m$ and $c$ with respect to the decimal logarithm of the reduced temperature $t$ vs $\log _{10} t$. All curves were obtained for $J_{A}=1$ and $J_{B}=-5$. If the functional dependence of these functions with respect to $t$ are similar to that in Eq. (3), with the corresponding critical exponent replacing $2-\alpha$, the derivatives drawn in the figures decouple the periodic from the critical behavior and quantify the critical parameters. For the first three functions it has been possible to evaluate both the critical exponent and the period of the oscillations assuming the dependence expressed by Eq. (3). We explored the critical region from $t \sim 10^{-3}$ down to $t \sim 10^{-9}$. For all fittings, errors are very small as indicated by $\chi^{2} \sim 10^{-6}$ or smaller.

$\xi\left(T>T_{c}\right)$ offers the most simple situation shown in Fig. 1. The function

$$
y(t)=a+\sum_{n=1}^{N} b_{n} \cos \left(n \omega t+\phi_{n}\right)
$$

is used to fit the data. When $N=1, \quad a=\nu=1.48432$ \pm 0.00029 , and $\omega=9.77151 \pm 0.00757$. Taking $N=2$, then $\nu=1.48431 \pm 0.00015$, and $\omega=9.77480 \pm 0.00401$, with $b_{2} / b_{1} \sim 0.06$, confirming that oscillations are nearly pure sinusoidal.

The zero-field magnetic susceptibility of hierarchical lattices is only well defined for $T<T_{c}$, as for $T>T_{c}$ it diverges with $h \rightarrow 0[19,20]$. So, both spontaneous magnetization and susceptibility shown in Figs. 2 and 3 are restricted to $T$ $<T_{c}$. It is clear from the figures, specially in the case of $\chi$, that a single harmonic is not sufficient to account for the oscillatory behavior. Thus, taking $N=2$ in Eq. (5), we have estimated $\gamma=2.83366 \pm 0.00027, \omega=9.77179 \pm 0.00175$, and $b_{2} / b_{1} \sim 0.49$. For $m$ we obtained $\beta=0.06708$ $\pm O\left(10^{-6}\right), \omega=9.77508 \pm 0.00901$ and $b_{2} / b_{1} \sim 0.22$. These ratios $b_{2} / b_{1}$ differ in the order of magnitude in comparison to that for $\xi$, and this is an evidence that the periodic functions $P_{x}(x=\xi, m, \chi)$ for $T>T_{c}$ and $T<T_{c}$ are distinct. The Table I collects the values of all fitting parameters for the three different functions.

Values in Table I for $\beta$ and $\nu$ are in great accordance with those of our former analysis, while the value for $\gamma$ has increased by some $4 \%$. This is due to the fact that we now evaluate both $m$ and $\chi$ at $h \equiv 0$, instead of in the (numerical) limit $h \rightarrow 0$. Using these values together with Rushbrook's equality we obtain, for $T<T_{c}, \alpha=-0,968 \cdots$. This is ex- 
actly the same value obtained for $\alpha^{\prime}$ in the region $T>T_{c}$, using the hyperscale relation and taking the fractal dimension of the lattice $d_{f}=2$.

The equality between $\alpha$ and $\alpha^{\prime}$ increases the confidence on the values for the other exponents. This becomes quite relevant in view of the curves shown in Figs. 4(a) and 4(b) for $\left|c(T)-c\left(T_{c}\right)\right|$. If we assume

$$
\begin{aligned}
& c(T)-c\left(T_{c}\right)=c_{1}\left(T_{c}-T\right)^{\alpha} P_{c}, \quad T<T_{c}, \\
& c\left(T_{c}\right)-c(T)=c_{1}^{\prime}\left(T-T_{c}\right)^{\alpha^{\prime}} P_{c}^{\prime}, \quad T>T_{c},
\end{aligned}
$$

where $P_{c}$ and $P_{c}^{\prime}$ indicate periodic functions, the curve clearly do not fit into the form (5), at least in the same $t$ interval used for the other three functions. For $T<T_{c}$ the maximum at $T_{m}$ restricts the size of the scaling region, while, for $T>T_{c}$, the presence of a second Schottky maximum may cause a similar effect.

For $T>T_{c}$ the oscillations have a somewhat clear single harmonic behavior, with decreasing amplitude, while $\alpha^{\prime}$ has not reached its constant value. For $T<T_{c}$ the value for $\alpha$ decreases rapidly with $t$, while the superimposed oscillations contain at least two harmonic contributions. To improve the results for $\alpha$ and $\alpha^{\prime}$ we have pushed the analysis down to $t \sim 10^{-11}$, the smallest value where the results are reliable. For $t$ smaller than this value, numerical fluctuations are relevant and the results become meaningless. Even so convergence has not been reached and it is imperative to include a richer form for the fitting function. The best results are obtained with the function

$$
y(t)=a+\frac{c}{t^{d}}+\frac{1}{t^{f}} \sum_{n=1}^{N} b_{n} \cos \left(n \omega t+\phi_{n}\right) .
$$

For $T<T_{c}$ we have $b_{2} / b_{1} \sim 1.5$, while, for $T>T_{c}$, $b_{2} / b_{1} \sim 0.12$. These results agree with our former indications that the periodic function $P$ changes at $T_{c}$ and that at least two relevant harmonic contributions are required for the oscillations in the regime $T<T_{c}$. The values obtained for the fitting parameters are listed on the Table I.

The best value for $\alpha$ agrees with the expected one, while $\alpha^{\prime}$ is still some $2 \%$ larger. However, the evaluation of the exponents at smaller and smaller neighborhood of $T_{c}$ indicates that these values should converge to $-0.968 \cdots$ if a region still closer to $T_{c}$ is explored.

Using the estimated value for $\Lambda_{c i c} \simeq 4.394 \cdots$ [21], we observe that all values for the frequency of oscillations reported above are bound to a small interval around $9.7747 \ldots$ predicted by Eq. (3), so that our results are in best accordance to those in Ref. [17]. To conclude, we observe that oscillations for $\chi$, when $T>T_{c}$ and $0 \neq h \ll 1$, are almost pure sinusoidal with $b_{2} / b_{1} \sim 0.1$. This completes the characterization of log-periodic oscillations for all thermodynamic functions, which is consistent with the identification of a discontinuous change for the oscillations below and above $T_{c}$.

The author is much indebted to Professor S. R. Salinas, S. T. R. Pinho, and T. A. S. Haddad for helpful discussions. The work was partially supported by $\mathrm{CNPq}$.
[1] U. Grimm and M. Baake, in The Mathematics of Long Range Aperiodic Order, edited by R. V. Moody (Kluwer Academic, Amsterdam, 1997), pp. 199-237.

[2] M. Queffélec, Substitutional Dynamical Systems-Spectral Analysis, Lecture Notes in Mathematics No. 1294 (SpringerVerlag, Berlin, 1987).

[3] A. Cobham, Math. Sys. Th. 6, 164 (1972).

[4] J. M. Luck, Europhys. Lett. 24, 359 (1993); J. Stat. Phys. 72, 417 (1993).

[5] S. T. R. de Pinho, T. A. S. Haddad, and S. R. Salinas, Braz. J. Phys. 27, 567 (1997).

[6] S. T. R. de Pinho, T. A. S. Haddad, and S. R. Salinas, Physica A 257, 515 (1998).

[7] A. C. N. de Magalhães, S. R. Salinas, and C. Tsallis, J. Phys. A 31, L567 (1998).

[8] A. N. Berker and S. Ostlund, J. Phys. C 12, 4961 (1979).

[9] R. B. Griffiths and M. Kaufman, Phys. Rev. B 24, 496 (1981).

[10] J. R. Melrose, J. Phys. A 16, 1041 (1983); 16, 3077 (1983).

[11] R. B. Griffiths and M. Kaufman, Phys. Rev. B 26, 5022
(1982); M. Kaufman and R. B. Griffiths, ibid. 30, 244 (1984).

[12] C. Tsallis and A. C. N. de Magalhães, Phys. Rep. 268, 305 (1996).

[13] P. E. Berché and B. Berché, J. Phys. A 30, 1347 (1997).

[14] D. Sornette, Phys. Rep. 297, 239 (1998).

[15] R. F. S. Andrade, Phys. Rev. E 59, 150 (1999).

[16] R. F. S. Andrade, Physica A 193, 29 (1993); Phys. Rev. B 48, 16095 (1993).

[17] T. A. S. Haddad, S. T. R. de Pinho, and S. R. Salinas, Phys. Rev. E 61, 3300 (2000).

[18] Th. Niemeijer and J. M. J. van Leeuwen, in Phase Transitions and Critical Phenomana, edited by C. Domb and M. S. Green (Academic, New York, 1976), Vol. 6.

[19] P. M. Bleher and E. Zalys, Commun. Math. Phys. 120, 409 (1989).

[20] M. Kaufman and R. B. Griffiths, J. Phys. A 15, L239 (1982).

[21] T. A. S. Haddad, MSc. thesis, Universidade de São Paulo, São Paulo, Brazil, 1999 (in Portuguese). 